\title{
Soluble fusion expression, characterization and localization of human $\beta$-defensin 6
}

\author{
AIJIE XIN ${ }^{1}$, YUE ZHAO ${ }^{4}$, HEGUO YU $^{3}$, HUIJUAN SHI $^{3}$, HAIYAN LIU $^{5}$, \\ HUA DIAO $^{3}$ and YONGLIAN ZHANG ${ }^{1-3}$

\begin{abstract}
${ }^{1}$ State Key Laboratory of Bioreactor Engineering, East China University of Science and Technology, Shanghai 200237;
${ }^{2}$ Shanghai Key Laboratory for Molecular Andrology, State Key Laboratory of Molecular Biology, Institute of Biochemistry and Cell Biology, Shanghai Institutes for Biological Sciences, Chinese Academy of Sciences, Shanghai 200031; ${ }^{3}$ NPFPC Key Laboratory of Contraceptives and Devices, Shanghai Institute of Planned Parenthood Research, Shanghai 200032; ${ }^{4}$ Reproductive Medical Center, Department of Obstetrics and Gynecology, Peking University Third Hospital, Beijing 100191; ${ }^{5}$ School of Life Science and Technology,
\end{abstract} \\ China Pharmaceutical University, Nanjing 210009, P.R. China
}

Received March 27, 2013; Accepted October 10, 2013

DOI: $10.3892 / \mathrm{mmr} .2013 .1768$

\begin{abstract}
Human $\beta$-defensin 6 (DEFB106) is an antimicrobial peptide expressed in the epididymis, testis and lung, which indicates that DEFB106 may be involved in innate immunity and fertility. However, as a $\beta$-defensin, this protein has not been well characterized. Using an intein-mediated fusion expression system, the recombinant DEFB106 was expressed and purified (yield, 3-5 mg/l) under optimized conditions. The purified protein was characterized using mass spectrometry and circular dichroism spectroscopy. The measured molecular weight was consistent with its theoretical value and the predominant secondary structure was $\beta$-sheet, the common structure of $\beta$-defensin family members. The purified DEFB106 showed antimicrobial activity against not only Escherichia coli (E. coli) and Candida albicans (C. albicans) SC5314, but also Staphylococcus aureus (S. aureus) CMCC26003. Furthermore, it exhibited a high affinity for heparin and lipopolysaccharide. In addition, it was determined that native DEFB106 was located in the epididymis, bone marrow and skin. These observations may aid in the determination of the physiological and pathological functions of DEFB106.
\end{abstract}

Correspondence to: Professor Yonglian Zhang, Shanghai Key Laboratory for Molecular Andrology, State Key Laboratory of Molecular Biology, Institute of Biochemistry and Cell Biology, Shanghai Institutes for Biological Sciences, Chinese Academy of Sciences, 320 Yueyang Road, Shanghai 200031, P.R. China E-mail: ylzhang@sibs.ac.cn

Dr Hua Diao, NPFPC Key Laboratory of Contraceptives and Devices, Shanghai Institute of Planned Parenthood Research, Shanghai 200032, P.R. China

E-mail: diaohua@gmail.com

Key words: human $\beta$-defensin 6, antimicrobial peptide, intein, purification, binding with heparin and lipopolysaccharide

\section{Introduction}

Defensins are cationic host defense polypeptides observed in numerous organisms, including plants $(1,2)$, invertebrates (3), vertebrates (4) and insects (5). The predominant functions of defensins serve to protect the host from the invasion of various pathogens. According to the spatial pattern of six conserved cysteine residues they are divided into $\alpha$-, $\beta$ - and $\theta$-defensin (6). $\beta$-defensins are widely distributed in a number of organs, particularly on the epithelial surfaces of male reproductive organs, suggesting they are involved in the resistance to microbial colonization and possibly in male fertility. Thus far, $>40$ putative $\beta$-defensins have been predicted by comprehensive searches of the human genome. However, due to their antibacterial activity, low molecular weight and the complicated disulfide bonds, few $\beta$-defensins have been expressed and purified in vitro.

Human $\beta$-defensin 6, also termed DEFB106, was initially identified and cloned by Yamaguchi et al in 2002 (7). Two copies of DEFB106 are located in 8p23-p22 with head-to-head orientation. The transcription of the DEFB106 gene was highly detected in the epididymis, testis and lung (7-9), and was downregulated in the epididymides of non-obstructive azoospermic men (10). Obtaining active DEFB106 peptide was an essential step in determining the involvement of DEFB106 in immunity and fertility. Although DEFB106 fused with thioredoxin A was expressed and purified in 2008 (11), the molecular weight, structure, and homogeneity of DEFB106 remained poorly characterized. In our previous study, several soluble $\beta$-defensins (e.g., HBD1-3 and rBin1b) were successfully expressed by a promising intein-mediated auto-cleavage expression system and the purified recombinant mature protein products were all bioactive with good homogeneity and structure $(12,13)$. This approach, not only improved the solubility and host cytotoxicity of defensins, but also omitted exogenous proteases required to remove the fusion tag. In the present study, the recombinant DEFB106 protein was expressed by the intein-mediated expression system and 
was well characterized. Purified DEFB106 peptides showed wide antimicrobial spectra against not only Escherichia coli (E. coli) and Candida albicans SC5314 (C. albicans), but also Staphylococcus aureus CMCC26003 (S. aureus) and demonstrated a high affinity for heparin and lipopolysaccharide (LPS). In addition, the tissue distribution of DEFB106 was determined by immunohistochemical staining (IHC).

\section{Materials and methods}

Plasmids and strains. pGEM-T Easy Vector systems (Promega Corporation, Madison, WI, USA) were utilized to clone DEFB106. pTWIN1 (New England Biolabs, Inc., Beijing, China) was used as the expression vector. E. coli BL21 (DE3) [F-ompT hsdSB (rB-mB)-gal dcm (DE3); Stratagene, La Jolla, CA, USA] was used as a host for heterologous protein expression. E. coli K12D31, S. aureus CMCC26003 and C. albicans SC5314 were used for antimicrobial assays.

Human tissue array and epididymis slice. Human tissue array was purchased from Shanxi Chaoying Biotechnology Co., Ltd., (Xi'an, China). The epididymis slice was preserved by our laboratory, and was obtained from a healthy donor (age, 25 years) who died in a car accident. All the procedures were approved by the Ethics Committee of Shanghai Institutes for Biological Sciences Chinese Academy of Sciences (Shanghai, China) and informed written consent was obtained from the donor's family.

Construction of expression vectors. The nucleotide sequence encoding the putative mature DEFB106 peptide containing 45 residues (FFDEKCNKLKGTCKNNCGKNE ELIALCQKSLKCCRTIQPCGSIID) was sub-cloned by PCR fromthepGEM-DEFB106plasmidand insertedintothepTWIN1 vector and digested with $\mathrm{NcoI}$ and XhoI (Fig. 1). The forward primer was 5'-GCGCCATGGACTTTTTTGATGAGAA-3', ( $N c o$ I restriction site underlined) and the reverse primer was 5'-GGCCTCGAGTTAATCTATAATGCTC-3' (XhoI restriction site underlined). Enzymes used in this study were purchased from Takara Co., Ltd. (Shiga, Japan).

Protein expression, purification and optimal $\mathrm{pH}$ for cleavage. All the experiments were conducted as in our previous study, but with minor modifications. (12). The vectors were confirmed with DNA sequencing and transformed into competent $E$. coli BL21 (DE3) cells. A single colony was incubated in $3 \mathrm{ml} \mathrm{LB}$ medium (Luria-Bertani broth) with $100 \mu \mathrm{g} / \mathrm{ml}$ ampicillin and cultured at $37^{\circ} \mathrm{C}$ and $83 \mathrm{x} \mathrm{g}$ in a shaking incubator overnight. The culture was transferred into $30 \mathrm{ml} \mathrm{LB}$ medium at a ratio of $2 \%(\mathrm{v} / \mathrm{v})$. When the cell cultures $\left(37^{\circ} \mathrm{C}, 83 \mathrm{x} \mathrm{g}\right)$ reached OD600 $\mathrm{nm}=0.8$, different concentrations of IPTG $(0.3,0.5$ and $1.0 \mathrm{mM}$ ) were added to induce protein expression at different temperatures $\left(37,22\right.$ and $\left.16^{\circ} \mathrm{C}\right)$. For a large scale culture, $250 \mathrm{ml} \mathrm{LB}$ medium was induced with $0.3 \mathrm{mM}$ IPTG at $\mathrm{OD} 600=0.8,37^{\circ} \mathrm{C}$. The cells were harvested by centrifugation $\left(5,000 \mathrm{x} \mathrm{g}\right.$ for $10 \mathrm{~min}$ at $\left.4^{\circ} \mathrm{C}\right)$ and were re-suspended in $5 \mathrm{ml}$ lysis buffer [20 mM sodium phosphate buffer, $\mathrm{pH} 8.5$, $0.5 \mathrm{M} \mathrm{NaCl}, 0.1 \mathrm{mM}$ EDTA and $0.1 \%$ Triton X-100 (v/v)] and lysed by sonication on ice. Following centrifugation at $13,000 \mathrm{x} g$ for $30 \mathrm{~min}$ the supernatant was isolated and incu- bated with $1 \mathrm{ml}$ chitin beads at $4^{\circ} \mathrm{C}$ for 30 min with gentle shaking. Subsequent to washes with 20 volumes of washing buffer (20 mM sodium phosphate buffer $\mathrm{pH} 8.0,0.5 \mathrm{M} \mathrm{NaCl}$, $0.1 \mathrm{mM}$ EDTA and $0.1 \%$ Triton X-100), beads affiliated with protein samples were equally divided into $1.5 \mathrm{ml}$ Eppendorf tubes, each containing $1.0 \mathrm{ml}$ cleavage buffer $(20 \mathrm{mM}$ sodium phosphate buffer, $0.5 \mathrm{M} \mathrm{NaCl}$ and $0.1 \mathrm{mM}$ EDTA) with varying $\mathrm{pHs}$ of 4.0, 4.5, 5.0, 5.5, 6.0, 6.5, 7.0 and 7.5. Following incubation overnight ( $>18 \mathrm{~h}$ ) at room temperature, beads were washed twice with cleavage buffer at the corresponding $\mathrm{pHs}$, and the supernatant was collected after centrifugation at $500 \mathrm{x}$ g for $5 \mathrm{~min}$. A total quantity of $10 \mu \mathrm{l}$ chitin beads were mixed with $10 \mu 12 \mathrm{X}$ sodium dodecyl sulphate-polyacrylamide gel electrophoresis (SDS-PAGE) loading buffer and bathed in boiling water for $10 \mathrm{~min}$. Cleavage efficiency was estimated by SDS-PAGE and the Gel Image system version 4.00 (Tanon Science and Technology Co., Shanghai, China). For large-scale preparation of DEFB106, the culture volume was expanded to 1 liter and $5 \mathrm{ml}$ beads were used. Following cleavage and elution, protein was concentrated by ultrafiltration with an Amicon Ultra 3K device (Millipore, Billerica, MA, USA) and applied to a Superdex-75 column by the Fast Protein Liquid Chromatography (FPLC) system (GE Healthcare, Wasukesha, WI, USA). Protein was eluted with $20 \mathrm{mM}$ phosphate buffer, $\mathrm{pH} 7.2$.

Mass spectrometry and circular dichroism spectroscopy analysis. The molecular weight of the purified protein was determined by matrix-assisted laser desorption ionization time-of-flight (MALDI-TOF) mass spectra recorded on a Bruker microFlex MALDI-TOF-MS spectrometer (ABI, Foster City, CA, USA). The peptide sample was mixed with an equal volume of matrix 2,5-dihydroxybenzoic acid. The instrument was operated in the positive ion/linear mode with an accelerating voltage of $20 \mathrm{kV}$ and scanning $\mathrm{m} / \mathrm{z}$ range of 3,000-12,000.

Circular dichroism spectroscopy was performed on a Jasco J-810 spectropolarimeter (Tokyo, Japan) using a quartz cell of $1 \mathrm{~mm}$ path length at $25^{\circ} \mathrm{C}$ with a scanning range of $200-250 \mathrm{~nm}$ and scanning speed of $100 \mathrm{~nm} / \mathrm{min}$.

Antimicrobial activity assays. The antimicrobial activity of DEFB106 was determined using the colony forming unit (CFU) assay, as described previously $(12,14)$. E. coli K12D31 grown in LB medium with $50 \mu \mathrm{g} / \mathrm{ml}$ streptomycin at $37^{\circ} \mathrm{C}$, S. aureus CMCC26003 grown in Mueller-Hinton (MH) broth at $37^{\circ} \mathrm{C}$ and $C$. albicans SC5314 grown in YPD (2\% tryptone, $1 \%$ yeast extract and $2 \%$ glucose, at $30^{\circ} \mathrm{C}$ ) were used as the sensitive strains. When grown to the mid-phase, the strains were diluted to a concentration of $10^{6} \mathrm{CFU} / \mathrm{ml}$ in the cold sterile $10 \mathrm{mM}$ sodium phosphate buffer (E. coli/S. aureus, pH 7.4; C. albican pH 6.8). When added to recombinant DEFB106 (10 mM PBS served as the negative control) of different concentrations, the strains were incubated for $3 \mathrm{~h}$ at $37^{\circ} \mathrm{C}$ (E. coli and S. aureus) and $30^{\circ} \mathrm{C}$ (C. albicans). The cultures were then serially diluted and cultured on LB (E. coli), MH (S. aureus) or YPD (C. albicans) agar plates in triplicate, respectively. Following incubation (E. coli and S. aureus at $37^{\circ} \mathrm{C}$ overnight, and $C$. albicans at $30^{\circ} \mathrm{C}$ for $24 \mathrm{~h}$ ), the surviving colonies were manually counted, and 
the antimicrobial activity was calculated using the formula: $\%$ survival $=$ (number of surviving colonies treatment with $\beta$-defensins/surviving colonies of control) x 100 (13).

Heparin and LPS-binding assays. Binding assays were performed in 96-well microplates at room temperature using the Octet Red 96 system (Fortebio, Menlo Park, CA, USA) $(15,16)$. Prior to executing the binding assay, the recombinant protein was labeled with biotin at a molar ratio of 1:1 (biotin:protein) in $20 \mathrm{mM}$ PBS at $\mathrm{pH} \mathrm{7.2,} \mathrm{and} \mathrm{the} \mathrm{unbound}$ biotin was removed by $\mathrm{Zeba}^{\mathrm{TM}}$ spin desalting columns (Thermo Fisher Scientific, Waltham, MA, USA) according to the manufacturer's instructions. In addition, Biosensor tips (Fortebio) were pre-wetted with PBS for $10 \mathrm{~min}$. Subsequent to this, $50 \mu \mathrm{g} / \mathrm{ml}$ DEFB106-biotin, PBS and heparin (GE Healthcare Bio-Sciences AB, Uppsala, Sweden) or lipopolysaccharide [LPS, from E. coli O111:B4 (Sigma-Aldrich, St. Louis, MO, USA)] with serial dilutions were added to the 96-well microtiter plates at a volume of $200 \mu \mathrm{l} /$ well. The subsequent measurement processes were all under computer control. Program procedures were established as follows: For the initial step, biosensors were washed in PBS buffer for $60 \mathrm{sec}$ to form a baseline; DEFB106 labeled with biotin was loaded into biosensors for $450 \mathrm{sec}$; biosensors were moved into PBS buffer in another line of wells for $420 \mathrm{sec}$ to remove any unbound DEFB106-biotin; the biosensors labeled with DEFB106-biotin were exposed to heparin or LPS of different concentrations for association, and were monitored for $300 \mathrm{sec}$; and then, the biosensors were moved back into PBS buffer to disassociate for another $300 \mathrm{sec}$. Data were fit globally and generated automatically by Octet User software (version 7.0; Fortebio).

To investigate whether the affinity of DEFB106 was based on its charges, $700 \mu \mathrm{g}$ purified protein was incubated with $700 \mu$ l Heparin Sepharose CL-6B (GE Healthcare, Wasukesha, WI, USA) in $20 \mathrm{mM}$ Tris- $\mathrm{HCl}, \mathrm{pH} 7.4$ at $4^{\circ} \mathrm{C}$ for $1 \mathrm{~h}$. After washing three times with $0.5 \mathrm{ml}$ buffer, heparin beads were divided into seven equal parts, and eluted by $\mathrm{NaCl}$ solution of different concentration ranging from 0 to $3.0 \mathrm{M}$. Then the beads were added to $100 \mu \mathrm{l}$ SDS-PAGE loading buffer and analysed on $15 \%$ SDS-PAGE gels.

IHC of DEFB106. IHC was performed with human tissue arrays and human epididymis tissue sections to identify the distribution of DEFB106. Primary goat antibody against the C-terminus of DEFB106 was purchased from Santa Cruz Biotechnology, Inc. (Santa Cruz, CA, USA) and diluted with PBS containing 10\% normal rabbit serum at a ratio of 1:50 and incubated with slices overnight at $4^{\circ} \mathrm{C}$. The slices were washed five times every $5 \mathrm{~min}$. The secondary antibody horseradish peroxidase-conjugated rabbit anti-goat (dilution 1:100; Boster Biological Technology, Ltd., Wuhan, China) was added and incubated with slices for $1 \mathrm{~h}$ at room temperature. The normal goat IgG served as a negative control. When stained with DAB (brown), the sections were counterstained with hematoxylin (blue).

\section{Results}

Expression and purification of recombinant DEFB106. The structure of the expression plasmid, pTWIN1 is shown in

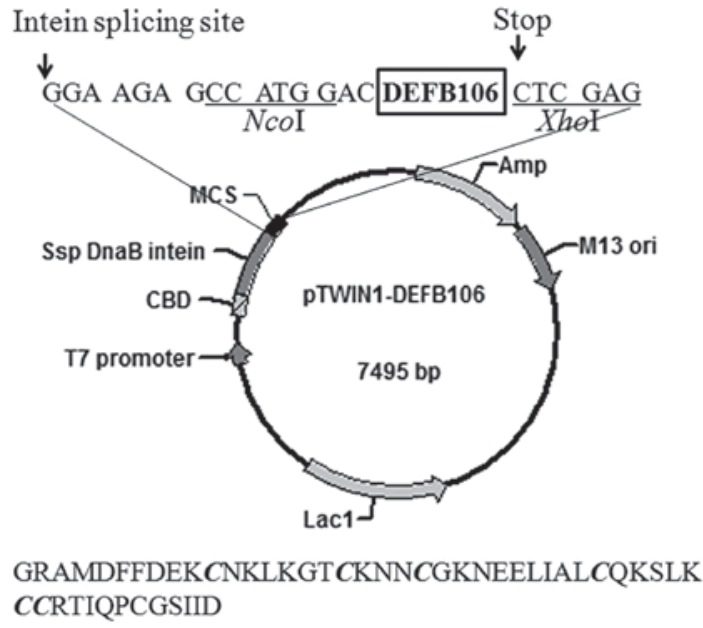

Figure 1. Schematic diagram of the recombinant vector pTWIN1-DEFB106. DNA sequence encoding the mature DEFB106 peptide is inserted between NcoI and XhoI restriction sites. The target peptide is fused with the C-terminal end of the Ssp intein peptide. The theoretical sequence of the released DEFB106 peptide is shown beneath the plasmid.
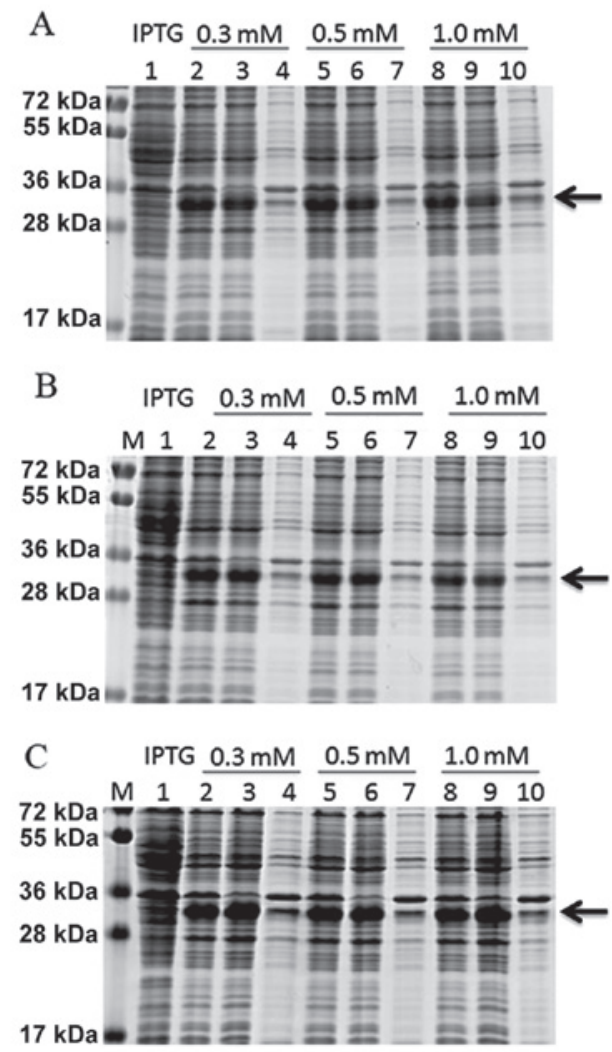

Figure 2. Sodium dodecyl sulfate-polyacrylamide gel electrophoresis of the expression levels of DEFB106 fusion proteins induced with different concentrations of IPTG at (A) $16^{\circ} \mathrm{C}$, (B) $22^{\circ} \mathrm{C}$ and (C) $37^{\circ} \mathrm{C}$. Lane 1 , whole cell lysate without IPTG induction as a negative control; lanes 2, 5 and 8 , whole cell lysate of cells induced with IPTG of different concentrations; lanes 3, 6 and 9 , supernatant of cell lysate following induction; lanes 4, 7 and 10, precipitation of cell lysate following induction. The arrows indicate the fusion protein CBD-intein-DEFB106.

Fig. 1. The nucleotide sequence encoding mature DEFB106 was sub-cloned and inserted into the C-terminus of $\mathrm{Ssp}$ DnaB intein. The amino acid sequence of the final eluted 
A
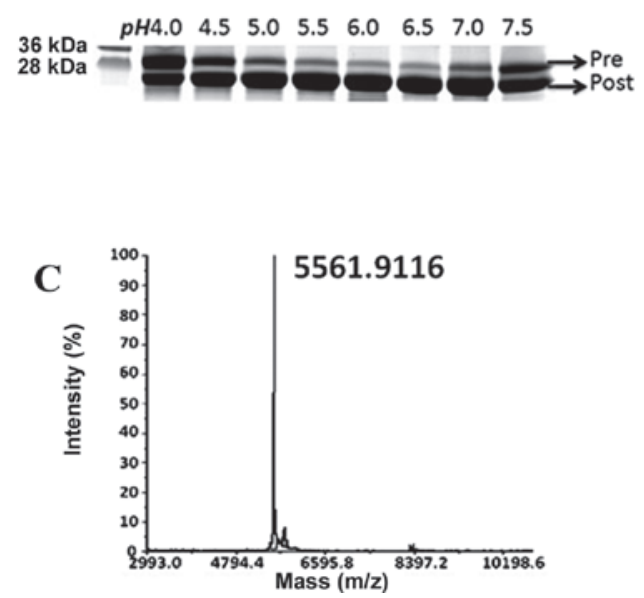

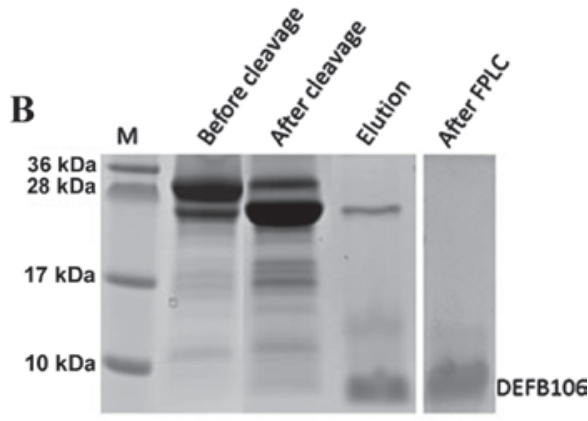

D

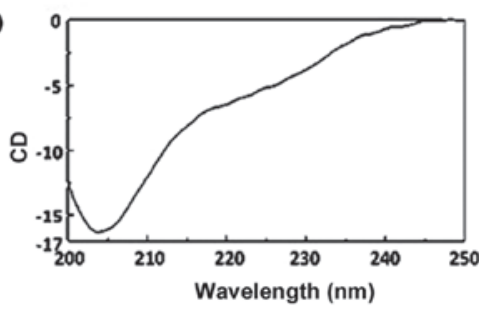

Figure 3. Optimization of auto-cleavage $\mathrm{pH}$ values and the purification and identification of DEFB106. (A) The most effective cleavage $\mathrm{pH}$ values were optimized by incubating the fusion protein in cleavage buffers with different $\mathrm{pH}$ ranging from 4.0 to 7.5. (B) Purification of recombinant DEFB106 protein by chitin affinity (chitin beads) and size-exclusion fast protein liquid chromatography chromatograms. (C) The mass spectrum shows the measured molecular weight of purified DEFB106 protein determined by mass spectrometry (matrix-assisted laser desorption ionization time-of-flight). (D) Circular dichroism spectra of the DEFB106 protein dissolved in $20 \mathrm{mM}$ phosphate buffer at $\mathrm{pH} 6.5$ with a final concentration of $0.2 \mathrm{mg} / \mathrm{ml}$. Pre, fusion protein prior to cleavage; post, fusion protein following cleavage.

polypeptide is shown beneath the plasmid map in Fig. 1. The first four amino acids, GRAM (glycine, arginine, alanine, methionine), were derived from pTWIN1 and the first glycine among them, adjacent to the intein, increased the cleavage efficiency of the fusion protein. In addition, one aspartate was added at the $\mathrm{N}$-terminum of the mature DEFB106 to avoid frame shift.

The concentration of IPTG and the temperature were optimized to maximize the expression of the DEFB106 fusion protein. SDS-PAGE analysis showed that the DEFB106 fusion protein was highly soluble independent of the IPTG concentration and culture temperature $\left(16,22\right.$ and $\left.37^{\circ} \mathrm{C}\right)$. The maximum expression yield was induced with $0.3 \mathrm{mM} \mathrm{IPTG}$ at $37^{\circ} \mathrm{C}$ (Fig. 2).

As the auto-cleavage mediated by intein is $\mathrm{pH}$ sensitive (17), it was determined that the optimal $\mathrm{pH}$ for auto-cleavage of intein-DEFB106 ranged from 6.0 to 6.5 (Fig. 3A). This finding was consistent with findings of previous studies, but different from that of other $\beta$-defensins, which range from $\mathrm{pH} 5.0$ to $6.0(12,13)$. The PAGE gel image analysis showed that $\sim 80 \%$ of DEFB106 fusion protein was cleaved (Fig. 3B). The purity of the released DEFB106 protein was determined by Tricine-SDS-PAGE (Fig. 3B), a useful tool for the analysis of small molecular proteins with different charges (18). Following one step affinity purification, the purity of DEFB106 was up to $80 \%$, and reached $95 \%$ with a yield of 3-5 mg/l after FPLC, analyzed by computer gray scan (Fig. 3B).

Identification of recombinant DEFB106. To analyze the quality of the recombinant protein, the molecular mass of the purified DEFB106 was identified by the MALDI-TOF mass spectrometry. As shown in Fig. 3C, the measured molecular mass was 5561.9, which was in accordance with the predicted theoretical molecular mass of 5562.6 Da. The predominant structure of

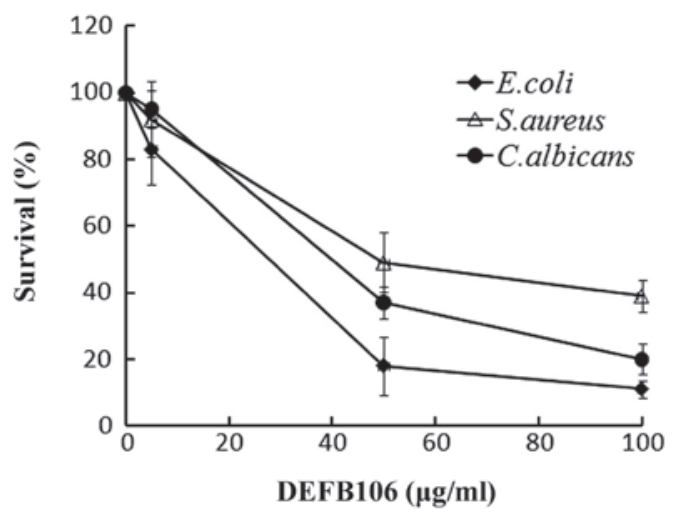

Figure 4. Antimicrobial activities of DEFB106 on model microbial strains. E. coli $\mathrm{K} 12 \mathrm{D} 31$ and $S$. aureus $\mathrm{CMCC} 26003$ were incubated in $10 \mathrm{mM}$ sodium phosphate buffer at $\mathrm{pH} 7.4$ and $37^{\circ} \mathrm{C}$ with exposure to DEFB106 addition for 3 h. C. albicans SC5314 was tested similarly at $\mathrm{pH} 6.8$ and $30^{\circ} \mathrm{C}$. Surviving bacteria were cultured, counted and the survival percentage was calculated. The three bacterial strains were suppressed with increasing concentrations of DEFB106 protein.

DEFB106 in $20 \mathrm{mM}$ phosphate buffer at $\mathrm{pH} 6.5$ was estimated by Circular dichroism spectra (Fig. 3D) to be $\beta$-sheet (approximately accounted for $66.5 \%$ ), which conformed to the typical $\beta$-sheet structure of $\beta$-defensins.

Antimicrobial activity. A broad spectrum of antimicrobial activity is a common feature of active $\beta$-defensins. The antimicrobial activity of the purified DEFB106 against $E$. coli K12D31, S. aureus and C. albicans was determined. The growth of all three bacterial strains was suppressed with increasing concentrations of DEFB106 protein (Fig. 4). E. coli $\mathrm{K} 12 \mathrm{D} 31\left(\mathrm{LD}_{50}=30 \mu \mathrm{g} / \mathrm{ml}\right)$ exhibited increased sensitivity to DEFB106 compared with C. albicans $\left(\mathrm{LD}_{50}=50 \mu \mathrm{g} / \mathrm{ml}\right)$ and S. aureus $\left(\mathrm{LD}_{50}=60 \mu \mathrm{g} / \mathrm{ml}\right)$. 

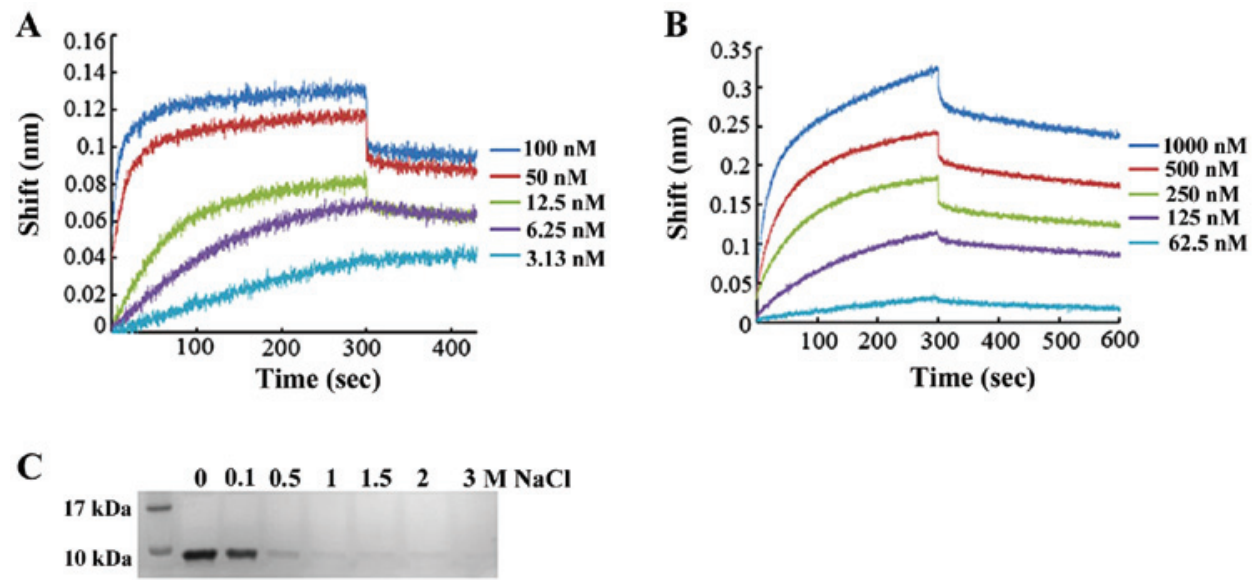

Figure 5. Association/dissociation kinetics and affinity of DEFB106 for heparin and LPS determined by Octet. APS sensors are coated with $50 \mu \mathrm{g} / \mathrm{ml}$ DEFB106 and associated with serially diluted (A) heparin and (B) LPS ranging from 100 and $1000 \mathrm{nM}$, respectively. (C) Specific affinity of DEFB106 for heparin beads was confirmed by the elution of DEFB106 (700 $\mu \mathrm{g}$ ) bound to heparin beads with elution buffers containing variant concentrations of $\mathrm{NaCl}$ and analyzed on a $15 \%$ SDS-PAGE gel.LPS, lipopolysaccharide.

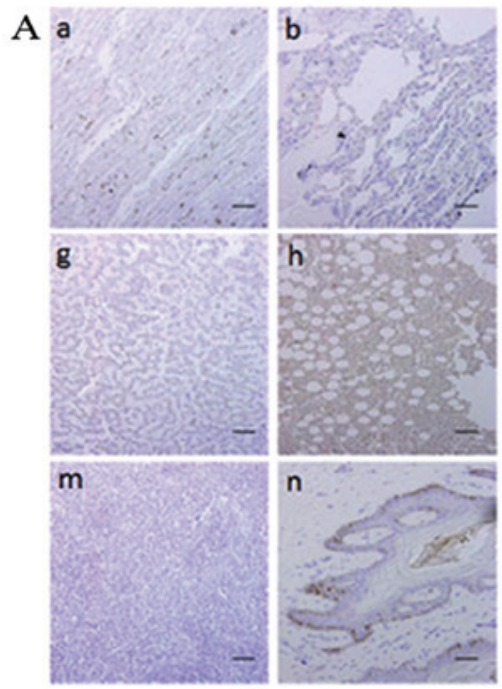

Cap

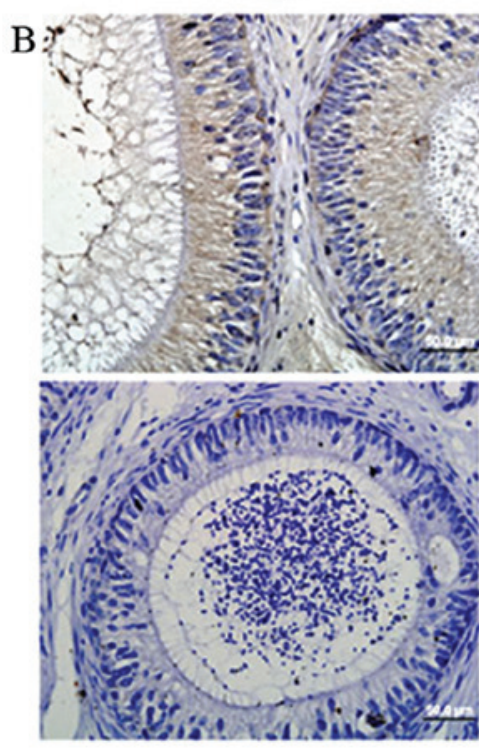

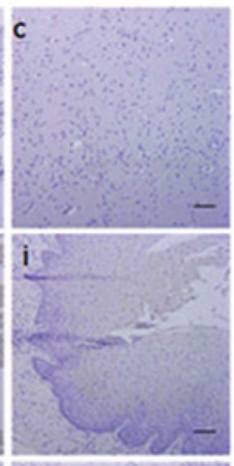

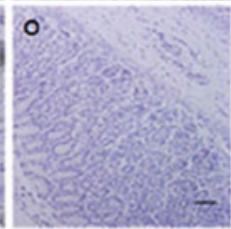

Cor
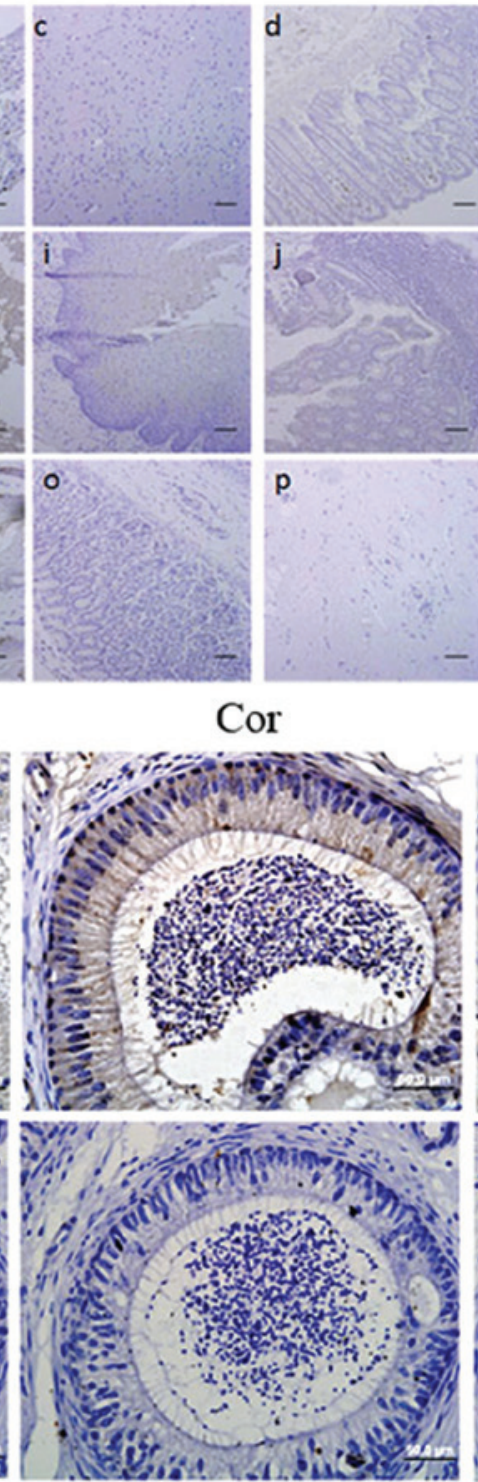
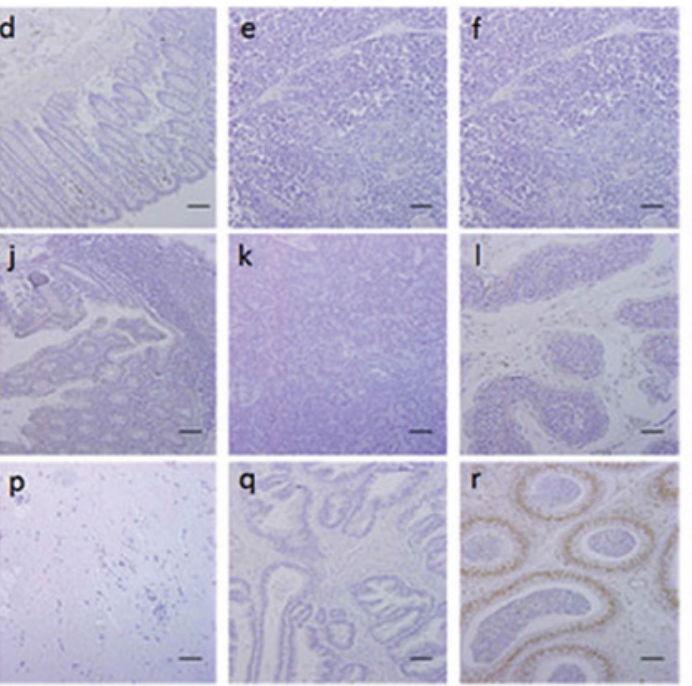

Cau

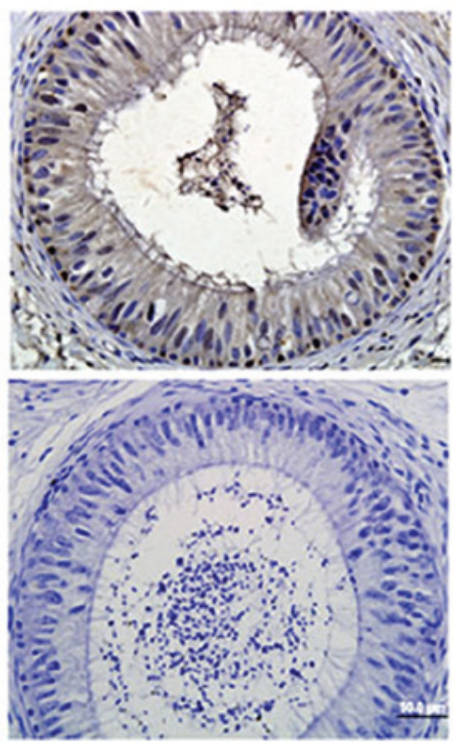

\section{DEFB106}

\section{Control}

Figure 6. Distribution of native DEFB106 determined by immunohistochemistry. (A) Human tissue arrays (scale bar, $200 \mu \mathrm{m}$ ). (Aa) Heart, (Ab) lung, (Ac) brain, (Ad) rectum, (Ae) thymus, (Af) ovary, (Ag) liver, (Ah) bone marrow, (Ai) esophagus, (Aj) colon, (Ak) pancreas, (Al) testis, (Am) spleen, (An) skin, (Ao) stomach, (Ap) fibrous connective tissue, (Aq) prostate and (Ar) epididymis. (B) Human epididymis paraffin sections (bars, $50 \mu \mathrm{m}$ ). Brown indicates DEFB106 signal and blue counterstained with hematoxylin shows the nuclei. Cap, caput; Cor, corpus; Cau, cauda. 
Affinity of DEFB106 for heparin and LPS. Certain antimicrobial peptides have been shown to bind heparin and LPS (19). Moreover, it has been hypothesized that the affinity of several $\beta$-defensins and related peptides for heparin-derived disaccharide may be correlated with their antimicrobial activity (20). As defensins are cationic peptides, whereas heparin and LPS have negative charges, there may be high affinity between peptides and polysaccharides. The affinity of DEFB106 for heparin and LPS was measured based on the Bio-layer interferomertry (BLI) instrument (Octet; ForteBio). To deduce the binding affinity via kinetic rate constants $\left(\mathrm{K}_{\mathrm{D}}=\mathrm{k}_{\text {off }} / \mathrm{k}_{\mathrm{on}}\right.$, where $\mathrm{K}_{\mathrm{D}}$ is the equilibrium dissociation constant, $\mathrm{k}_{\text {on }}$ the association rate constant, and $\mathrm{k}_{\text {off }}$ the dissociation rate constant), biosensor arrays were loaded with $50 \mu \mathrm{g} / \mathrm{ml}$ DEFB106-biotin and moved to heparin (concentrations ranged from 100 to $3.125 \mathrm{nM}$ ) or LPS (concentrations ranged from 1,000 to $62.5 \mathrm{nM}$ ), respectively. As shown in Fig. 5A and B, the affinity of DEFB106 binding with heparin and LPS was determined to be $\mathrm{K}_{\mathrm{D}}=5.08 \mathrm{E}-11 \mathrm{M}$ and $\mathrm{K}_{\mathrm{D}}=1.90 \mathrm{E}-08 \mathrm{M}$. The affinity of DEFB106 for heparin was higher than that for LPS. The affinity of DEFB106 protein for heparin beads was also approved by its binding and elution from heparin beads with $\mathrm{NaCl}$ solution as shown in Fig. 5C, which demonstrated that the negative charges may be predominantly responsible for the affinity.

Localization of DEFB106 by IHC. The localization of native DEFB106 protein was important in the investigation of the physiological and pathological functions. By using an antibody that recognized the C-terminus of DEFB106, IHC was used to identify the distribution of DEFB106 protein on human tissue arrays. Notably, native DEFB106 signals were observed in the bone marrow and skin, and particularly in the nuclei of epididymal cells (Fig. 6). In addition, DEFB106 signals in the skin appeared to be in the nuclei of hair follicle peripheral cells. As the high intensity of DEFB106 signals in the epididymis were consistent with the high mRNA level, IHC of human epididymis tissue slides were further stained to analyze the distribution of DEFB106. The positive signals were detected through the whole tissue from caput to cauda regions. The signals in the cauda region were more intensive than in the caput and corpus.

\section{Discussion}

Currently, only 13 protein products have been confirmed among $>40$ open reading frames (ORFs) of putative human $\beta$-defensins. Using the intein-mediated auto-cleavage expression system, we not only obtained active DEFB106 protein with high yield, but also successfully expressed and purified numerous $\beta$-defensins, including Bin1b, HBD1, HBD2 and HBD3 $(12,13)$. This system allowed easy purification under mild conditions (21) with high yield and low cost, which renders it an option for the investigation of other human defensins.

Although the recombinant expression of DEFB106 protein has been suggested (11), the protein has not been well characterized. In the present study, the molecular weight of the predominant peak of recombinant DEFB106 protein recorded by mass spectrometry, was consistent with its calculated value. In addition, the major structure of the recombinant DEFB106 protein in solution was the $\beta$-sheet, which was in accordance with the common features of other $\beta$-defensins. The DEFB106 protein produced by the intein-mediated auto-cleavage system showed a wide antimicrobial spectrum (including E. coli K12D31, S. aureus and C. albicans), similar with that of other $\beta$-defensins including Bin1b (22), Defb15 (23), HBD1, HBD2 and HBD3 (24). However, Huang et al (11) demonstrated that purified DEFB106 showed antimicrobial activity against $E$. coli but not $S$. aureus. The difference may derive from the different quality of proteins and secondary structures in solution.

DEFB106 showed novel interaction with heparin with high affinity, suggesting its possible involvement in innate and adaptive immunity. Heparin is a highly sulfated glycosaminoglycan (GAG), produced in high abundance by mammalian cells and has the highest negative charge density. By interacting with heparin, defensin appears to be involved in neutralizing the heparin anticoagulation activity and the inhibition activity of non-enzymatic fibrinolysis (25). Additionally, defensins are involved in the release of GAGs close to the sites of bacterial pathogen infection, suggesting that they are involved in innate immunity (20). As with chemokine/glycosaminoglycan (GAG) interactions, defensin-GAG complexes are likely to be important in chemotaxis (26). In addition, $\beta$-defensins have been shown to link innate and adaptive immune systems (27). For example, HBD1-4 possesses chemotactic properties by recruiting immature dendritic cells, memory $\mathrm{T}$ cells and/or mast cells (28-30). As a novel human $\beta$-defensin, DEFB106 may serve as an important component in local tissues in combating infectious pathogens and inhibiting non-enzymatic fibrinolysis.

DEFB106 interacted with LPS, a predominant component of the surface of Gram-negative bacteria and led to bacterial death, possibly by disrupting the lipid bilayers as demonstrated in previous studies (31-33). As antimicrobial peptides are known to bind with the negatively charged LPS-leaflet of Gram-negative bacteria and studies have shown that $\beta$-defensin has the potential to neutralize LPS-induced immune responses (34-36), DEFB106 may also participate in regulating the inflammatory response.

Mammalian $\beta$-defensins undergo rapid evolution and in certain cases orthologous genes evolve to adapt to species-specific functions. Native DEFB106 was localized in the nuclei of epididymis, bone marrow and skin, which was inconsistent with its orthologous gene Defb15 $(7,23)$. This finding suggests that DEFB106 may have other unknown functions in addition to antimicrobial activity.

In conclusion, recombinant DEFB106 was prepared and characterized with broad antimicrobial spectrum and high affinity for heparin and LPS. The distribution of native DEFB106 was determined predominantly in the nuclei of epididymal cells, bone marrow and skin. The results of the present study may therefore aid in determining the physiological and pathological functions of DEFB106.

\section{Acknowledgements}

The authors greatly acknowledge Professor Xiangfu Wu (Chinese Academy of Sciences, China) for the provision of E. coli K12D31, professor Guanghua Huang (Chinese Academy of Sciences, China) for C. albicans and professor YuanKang Ye (Tongji Hospital, China) for S. aureus, and also thank Aihua Liu for technical assistance in tissue section preparation. 
This study was supported by grants from the Natural Science Foundation of China (project nos. 31101030, 81270744 and 30930053) and the Chinese Academy of Sciences [Knowledge Innovation Program Grant (grant no. KSCX2-EW-R-07)].

\section{References}

1. Thomma BP, Cammue BP and Thevissen K: Plant defensins Planta 216: 193-202, 2002.

2. Lay FT and Anderson MA: Defensins - components of the innate immune system in plants. Curr Protein Pept Sci 6: 85-101, 2005.

3. Rodríguez de la Vega RC and Possani LD: On the evolution of invertebrate defensins. Trends Genet 21: 330-332, 2005.

4. Lehrer RI and Ganz T: Defensins of vertebrate animals. Curr Opin Immunol 14: 96-102, 2002.

5. Hoffmann JA and Hetru C: Insect defensins: inducible antibacterial peptides. Immunol Today 13: 411-415, 1992.

6. Tang YQ, Yuan J, Osapay G, et al: A cyclic antimicrobial peptide produced in primate leukocytes by the ligation of two truncated alpha-defensins. Science 286: 498-502, 1999.

7. Yamaguchi Y, Nagase T, Makita R, et al: Identification of multiple novel epididymis-specific beta-defensin isoforms in humans and mice. J Immunol 169: 2516-2523, 2002.

8. Kao CY, Chen Y, Zhao YH and Wu R: ORFeome-based search of airway epithelial cell-specific novel human $\beta$-defensin genes. Am J Respir Cell Mol Biol 29: 71-80, 2003.

9. Semple CA, Rolfe M and Dorin JR: Duplication and selection in the evolution of primate beta-defensin genes. Genome Biol 4 R31, 2003

10. Dubé E, Hermo L, Chan PT and Cyr DG: Alterations in gene expression in the caput epididymides of nonobstructive azoospermic men. Biol Reprod 78: 342-351, 2008.

11. Huang L, Ching CB, Jiang R and Leong SS: Production of bioactive human beta-defensin 5 and 6 in Escherichia coli by soluble fusion expression. Protein Expr Purif 61: 168-174, 2008

12. Diao H, Guo C, Lin D and Zhang Y: Intein-mediated expression is an effective approach in the study of beta-defensins. Biochem Biophys Res Commun 357: 840-846, 2007.

13. Dong J, Yu H, Zhang Y, Diao H and Lin D: Soluble fusion expression and characterization of human beta-defensin 3 using a novel approach. Protein Pept Lett 18: 1126-1132, 2011.

14. Yenugu S, Chintalgattu V, Wingard CJ, Radhakrishnan Y, French FS and Hall SH: Identification, cloning and functional characterization of novel beta-defensins in the rat (Rattus norvegicus). Reprod Biol Endocrinol 4: 7, 2006.

15. Abdiche Y, Malashock D, Pinkerton A and Pons J: Determining kinetics and affinities of protein interactions using a parallel real-time label-free biosensor, the Octet. Anal Biochem 377: 209-217, 2008.

16. Do T, Ho F, Heidecker B, Witte K, Chang L and Lerner L: A rapid method for determining dynamic binding capacity of resins for the purification of proteins. Protein Expr Purif 60: 147-150, 2008.

17. Mathys S, Evans TC, Chute IC, et al: Characterization of a self-splicing mini-intein and its conversion into autocatalytic $\mathrm{N}$ - and C-terminal cleavage elements: facile production of protein building blocks for protein ligation. Gene 231: 1-13, 1999.

18. Schägger $\mathrm{H}$ and von Jagow $\mathrm{G}$ : Tricine-sodium dodecyl sulfate-polyacrylamide gel electrophoresis for the separation of proteins in the range from 1 to $100 \mathrm{kDa}$. Anal Biochem 166: 368-379, 1987
19. Andersson E, Rydengård V, Sonesson A, Mörgelin M, Björck L and Schmidtchen A: Antimicrobial activities of heparin-binding peptides. Eur J Biochem 271: 1219-1226, 2004.

20. McCullough BJ, Kalapothakis JM, Chin W, et al: Binding a heparin derived disaccharide to defensin inspired peptides: insights to antimicrobial inhibition from gas-phase measurements. Phys Chem Chem Phys 12: 3589-3596, 2010.

21. Banki MR, Feng L and Wood DW: Simple bioseparations using self-cleaving elastin-like polypeptide tags. Nat Methods 2: 659-661, 2005.

22. Guo C, Diao H, Lian Y, et al: Recombinant expression and characterization of an epididymis-specific antimicrobial peptide BIN1b/SPAG11E. J Biotechnol 139: 33-37, 2009.

23. Zhao Y, Diao H, Ni Z, Hu S, Yu H and Zhang Y: The epididymis-specific antimicrobial peptide $\beta$-defensin 15 is required for sperm motility and male fertility in the rat (Rattus norvegicus). Cell Mol Life Sci 68: 697-708, 2011.

24. Pazgier M, Hoover DM, Yang D, Lu W and Lubkowski J: Human beta-defensins. Cell Mol Life Sci 63: 1294-1313, 2006.

25. Liapina LA, Kondashevskaia MV, Kokriakov VN and Shamova OV: Interaction of heparin with defensin, a nonenzymatic cationic protein from neutrophils. Vopr Med Khim 38: 39-42, 1992 (In Russian).

26. Seo ES, Blaum BS, Vargues T, et al: Interaction of human $\beta$-defensin 2 (HBD2) with glycosaminoglycans. Biochemistry 49: 10486-10495, 2010.

27. Yang D, Chertov O, Bykovskaia SN, et al: Beta-defensins: linking innate and adaptive immunity through dendritic and T cell CCR6. Science 286: 525-528, 1999.

28. Chen X, Niyonsaba F, Ushio H, et al: Antimicrobial peptides human beta-defensin (hBD)-3 and hBD-4 activate mast cells and increase skin vascular permeability. Eur J Immunol 37: 434-444, 2007.

29. Hoover DM, Boulegue C, Yang D, et al: The structure of human macrophage inflammatory protein-3alpha/CCL20. Linking antimicrobial and $\mathrm{CC}$ chemokine receptor-6-binding activities with human beta-defensins. J Biol Chem 277: 37647-37654, 2002.

30. Vargues T, Morrison GJ, Seo ES, et al: Efficient production of human beta-defensin 2 (HBD2) in Escherichia coli. Protein Pept Lett 16: 668-676, 2009.

31. Ouellet M, Otis F, Voyer N and Auger M: Biophysical studies of the interactions between 14-mer and 21-mer model amphipathic peptides and membranes: insights on their modes of action. Biochim Biophys Acta 1758: 1235-1244, 2006.

32. Shai Y: Mechanism of the binding, insertion and destabilization of phospholipid bilayer membranes by alpha-helical antimicrobial and cell non-selective membrane-lytic peptides. Biochim Biophys Acta 1462: 55-70, 1999.

33. Buffy JJ, Hong T, Yamaguchi S, Waring AJ, Lehrer RI and Hong M: Solid-state NMR investigation of the depth of insertion of protegrin-1 in lipid bilayers using paramagnetic $\mathrm{Mn}^{2+}$. Biophys J 85: 2363-2373, 2003.

34. Motzkus D, Schulz-Maronde S, Heitland A, et al: The novel beta-defensin DEFB123 prevents lipopolysaccharide-mediated effects in vitro and in vivo. FASEB J 20: 1701-1702, 2006.

35. Liu H, Yu H, Gu Y, et al: Human beta-defensin DEFB126 is capable of inhibiting LPS-mediated inflammation. Appl Microbiol Biotechnol 97: 3395-3408, 2013.

36. Yu H, Dong J, Gu Y, Liu H, Xin A, et al: The novel human $\beta$-defensin 114 regulates lipopolysaccharide (LPS)-mediated inflammation and protects sperm from motility loss. J Biol Chem 288: 12270-12282, 2013. 